\title{
Tripronuclear Zygotes in IVF Laboratory Quality Control: Experimental Evaluation and Potential Applications
}

\author{
Suzhu Chen*, Pengyu Huang*, Yan Sun*, Shengrong Du, Yunhong Lin, Beihong Zheng, Dianliang Lin \\ Reproductive Medicine Center, Fujian Maternity and Child Health Hospital, Affiliated Hospital of Fujian Medical University, Fuzhou, 35000I, People's \\ Republic of China \\ *These authors contributed equally to this work \\ Correspondence: Beihong Zheng; Dianliang Lin, Tel +86-1376382I254, Email zhengbeihong20I0@I63.com; mqld|@I63.com
}

\begin{abstract}
This study set out to evaluate quality control within a new in vitro fertilization (IVF) laboratory environment and of new incubators based on the culture results of tripronuclear zygotes. The representative environmental indicators within new and old IVF laboratories were monitored, and tripronuclear zygotes were cultured in the two laboratories; the results were analyzed and compared. Subsequently, tripronuclear (3PN) zygotes were cultured in both new and old incubators and the culture results were compared. No differences were found in embryo development between 3PN zygotes in the old and new laboratories. However, in the quality control test, the degeneration rate and developmental arrest rate in the new incubator early phase group were significantly increased when compared with the old incubators. Moreover, the grade I embryo rate also decreased significantly. Nevertheless, all the above comparisons in the new incubator later phase group showed no statistical significance as compared to those observed in old incubators. Tripronuclear zygotes are sensitive to the environment in IVF laboratories and can be considered useful during quality control trials of new IVF laboratories and new equipment including incubators.
\end{abstract}

Keywords: embryo development, new incubator, degeneration rate, developmental arrest rate

\section{Introduction}

The IVF laboratory plays a central role in assisted reproductive technology (ART), which directly affects final pregnancy outcome. Therefore, quality control within the IVF laboratory is essential, ${ }^{1}$ and is even more critical for new laboratories and new equipment. ${ }^{2}$ Before a new IVF laboratory is put into use, quality tests of its environment, instruments, various consumables, and reagents must take place. ${ }^{3}$ ART should only be conducted if all tests are passed. Mouse embryo tests are usually carried out before a new laboratory is used for clinical cases. The mouse embryo test is the industry standard for quality control, as it is reported on nearly all certificates of analysis, yet neither the assay's endpoint nor the conditions are standardized. The most common endpoint reported with the mouse embryo test is blastocyst formation at $96 \mathrm{~h}$ of culture. If $>80 \%$ of the fertilized oocytes have reached the blastocyst stage, the tested laboratory or device is considered suitable for clinical use. ${ }^{4}$ Although mouse embryo tests have a role in clinical IVF, it is at best a test of the developmental ability of mouse embryos. There is no guarantee that factors that do not affect mice will not be harmful to humans. The ultimate quality control for all mediators of clinical IVF is the ability to support the growth of human embryos. However, it is clearly unethical to use normal human embryos for this purpose. In addition, mouse embryo tests require the breeding and housing of mice and the complicated process of ovarian stimulation. ${ }^{5}$ The absence of an animal room is an additional difficulty in some reproductive centers. A new simple and effective method is therefore needed. Quality control aims to ensure that human embryos can divide and develop normally under culture conditions in IVF laboratories. During ART some untransferable, abnormal embryos such as tripronuclear (3PN) zygotes are produced, which still have the ability to divide. ${ }^{6}$ By trialing the use of $3 \mathrm{PN}$ zygotes 
during quality control within new laboratories and incubators, our study offers a new, practical, and tested method for quality control in IVF laboratories; it has been named the human tripronuclear (3PN) zygote biological test.

\section{Materials and Methods}

\section{Case Selection and Grouping}

Cases were selected and grouped according to the following criteria among patients treated with IVF-ET in old and new laboratories at our center. An "old laboratory" indicates that, after passing the expert review of the Health Commission, it can be officially run and achieve a stable success rate. "New laboratory" refers to a new laboratory that has passed the routine air quality test used during the pre-clinical quality control phase. The criteria included were: (1) females 22-35 y; (2) IVF cycle; (3) 3PN at 16-18 h after fertilization; (4) normal cleavage of the same batch of embryos; (5) no history of hereditary disease. After screening, 634 3PN zygotes were obtained for further use. Among them, 164 were cultured in old incubators (Geri, Serono, German) in the old laboratory, 168 in old incubators in the new laboratory, 150 in new incubators (Geri, Serono) with $<2$ months of use in the new laboratory, and 152 in new incubators $>2$ months of use in the new laboratory. Tripronuclear zygotes that met the above criteria were collected in our laboratory and randomly grouped to avoid bias.

\section{IVF Procedures}

Administration of human chorionic gonadotropin (hCG) was performed when the female patients met the following criteria: 1) the total duration of gonadotropin ( $\mathrm{Gn}$ ) administration was $>7 \mathrm{~d} ; 2$ ) all patients being treated in new and old laboratories met the following criteria; 3) E2 levels per preovulatory follicle ( $>14 \mathrm{~mm}$ ) were $200-300 \mathrm{pg} / \mathrm{mL}$; 4) the ratio of follicles $(>18 \mathrm{~mm})$ to preovulatory follicles $(>14 \mathrm{~mm})$ was $60 \%-70 \%$; or the ratio of follicles $(>20 \mathrm{~mm})$ to preovulatory follicles $(>14 \mathrm{~mm})$ was $40 \%-50 \%$. The patients were injected with $5000-10,000 \mathrm{IU}$ of $\mathrm{hCG}$, and the oocytes were retrieved $36-38 \mathrm{~h}$ later. After $3 \mathrm{~h}$, IVF procedures were performed with 100,000-200,000 motile spermatozoa per $\mathrm{mL}$ for 5-10 oocytes, which were then cultured in fertilization media (IVF, Vitrolife) in incubators (Geri, Serono) at $37{ }^{\circ} \mathrm{C}$ with $89 \% \mathrm{~N}_{2}, 6 \% \mathrm{CO}_{2}$, and $5 \% \mathrm{O}_{2}$. The embryos were cultured until Day 3 in global culture media (Lifeglobal, USA) after removal of granulosa cells. An embryologist observed whether 3PN were present at 16-18 $\mathrm{h}$ after fertilization. All 3PN zygotes were evaluated using the grading systems developed by Veeck (1986) for determining cleavage stage.

\section{Embryonic Development Index}

Cleavage rate $=$ the number of cleavage/total number of fertilized zygotes. Grade I embryo rate $=$ the number of grade I embryos/the total number of fertilized zygotes. Degradation rate $=$ the number of degraded embryos/the total number of fertilized zygotes. Arrest rate $=$ the number of development-arrested embryos/the total number of fertilized zygotes.

\section{Measurement of Environmental Factors}

An Environmental Test Meter (AdvancedSense, UK) was used to measure temperature, humidity, and volatile organic compound (VOC) concentration in the IVF laboratory. A handheld detector (Handheld 3016 IAQ) was used to determine PM2.5 levels. The measurement was taken during each embryology procedure and the mean value of three measurements was obtained.

\section{Statistical Analysis}

SPSS v.20.0 software was used for statistical analysis. Continuous variables were expressed as "mean \pm SD" and $95 \%$ confidence interval (CI) ( $t$-test), while the categorical variables were expressed as n (\%) and $95 \% \mathrm{CI}\left(\chi^{2}\right.$-test). ANOVA was used for group comparisons of parametric data sets. 
Table I Environment Quality Test

\begin{tabular}{|l|c|c|c|}
\hline Index & Old Laboratory Group $(\mathbf{n}=\mathbf{1 8 3})$ & New Laboratory Group (n = 185) & P-value \\
\hline Temperature $\left({ }^{\circ} \mathrm{C}\right)$ & $24.09 \pm 0.53$ & $24.18 \pm 0.89$ & 0.240 \\
Humidity $(\%)$ & $50.11 \pm 8.22$ & $49.25 \pm 7.40$ & 0.292 \\
PM2.5 $\left(\mathrm{pc} / \mathrm{m}^{3}\right)$ & $0.77 \pm 0.15$ & $0.75 \pm 0.19$ & 0.263 \\
VOC Concentration $(\mathrm{Ppb})$ & $264.10 \pm 53.60$ & $267.20 \pm 65.71$ & 0.620 \\
\hline
\end{tabular}

Abbreviations: $n$, measured days; VOC, volatile organic compounds; PM, fine particulate matter.

\section{Results}

\section{Environment Testing of the New and Old IVF Laboratories}

Temperature, humidity, and VOC concentration were tested three times a day, and the average value was taken as a sample value. As can be seen in Table 1, there was no significant difference in temperature, humidity, and PM2.5 between the two groups. The data indicated that the environment of the new laboratory was at a stable and ideal status.

\section{The in vitro Development of Human 3PN Zygotes}

During ART, occasionally two sperm may enter an oocyte to form a 3PN zygote, and 3PN embryos should be de-selected when choosing good-quality embryos. However, owing to their ability to divide, they can be used as an ideal experimental model. More importantly, they allow tests to be performed using human embryos without breaking ethical rules. As shown in Figure 1, some 3PN zygotes (Figure 1A and D) continued to develop to the cleavage stage on Day 2 (Figure 1B and E) and Day 3 (Figure 1C and F); however, some also underwent degradation or developmental arrest.

\section{Development of 3PN Zygotes in the New and Old Laboratories}

The new embryology laboratory at our center had passed its environment quality test and the mouse embryo biological test before being put into use. Data were collected from 164 3PN zygotes cultured for $3 \mathrm{~d}$ in the old incubators within the old laboratory and $1683 \mathrm{PN}$ zygotes cultured for $3 \mathrm{~d}$ in old incubators within the new laboratory. Data regarding their
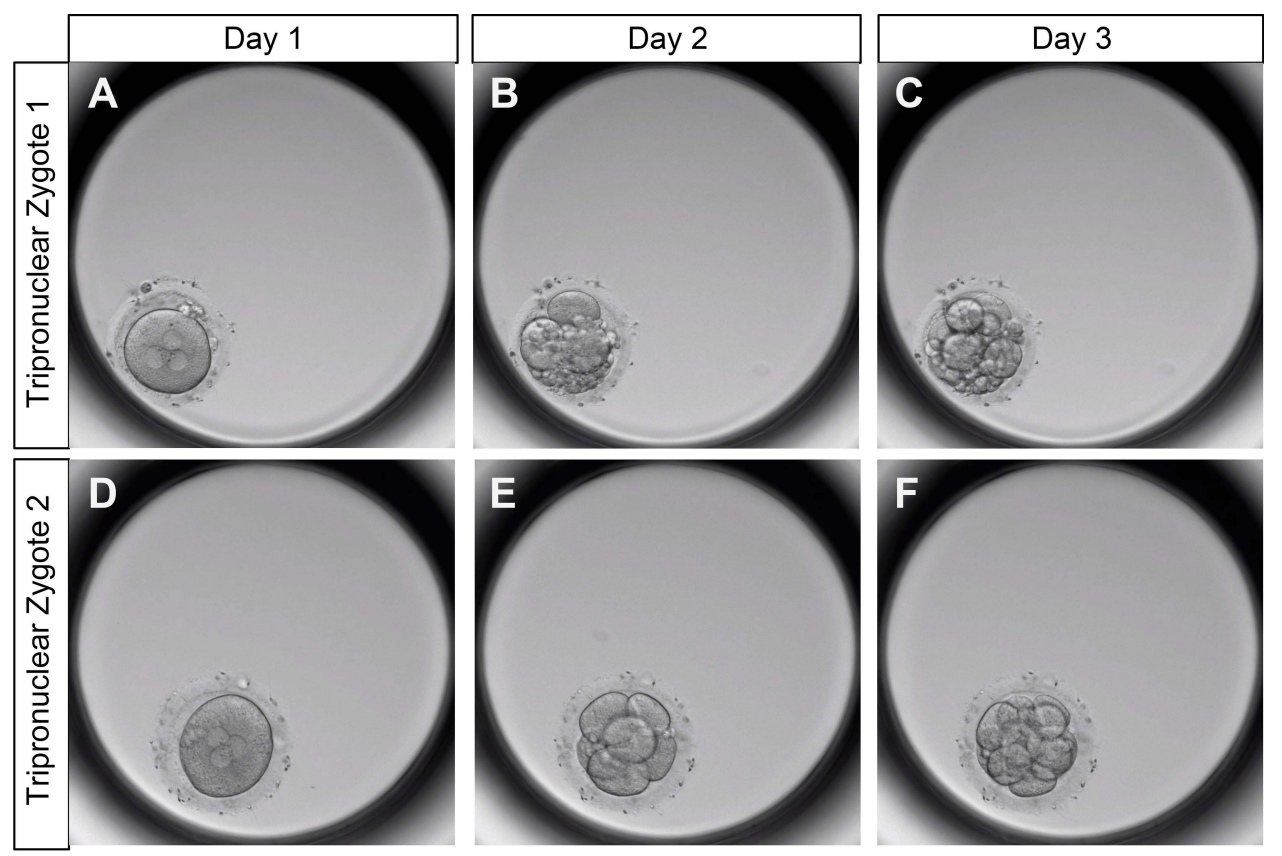

Figure I The in vitro development of human tripronuclear (3PN) zygotes. (A and D) 3PN zygotes on Day I. (B and E) 3PN zygotes continued to develop to the cleavage stage on Day 2. (C and F) 3PN zygotes continued to develop to the cleavage stage on Day 3. 
Table 2 Development Comparison of 3PN Zygotes in the New and Old Laboratories

\begin{tabular}{|c|c|c|c|}
\hline Index & $\begin{array}{l}\text { Old Laboratory } \\
\text { Group } \\
(n=164)\end{array}$ & $\begin{array}{c}\text { New Laboratory } \\
\text { Group } \\
(\mathbf{n}=168)\end{array}$ & P-value \\
\hline Cleavage rate & $100 \%(164 / 164)$ & $99.4 \%(167 / 168)$ & 0.969 \\
\hline Grade I embryo rate & $36.0 \%(59 / 164)$ & $40.5 \%(68 / 168)$ & 0.646 \\
\hline Degradation rate & $11.6 \%(19 / 164)$ & II.9\% (20/168) & 0.929 \\
\hline Development arrest rate & $3.0 \%(5 / 164)$ & $1.2(2 / 168)$ & 0.440 \\
\hline
\end{tabular}

developmental status were analyzed. The grade I embryo rate, the cleavage rate, and the development arrest rate were specifically analyzed. Our data showed that there was no significant difference in the cleavage rate, the grade I embryo rate, the degeneration rate, and the development arrest rate between the two groups (Table 2). These results suggested that the environment of the new laboratory met the requirements for embryo culture conditions, consistent with the previous environment quality test.

\section{Development of 3PN Zygotes in the New and Old Incubators}

Three groups were cultured in the new laboratory. The data of the new incubator early phase group, where $1503 \mathrm{PN}$ zygotes were cultured in new incubators with $<2$ months of use, and of the new incubator later phase group, where 152 3PN zygotes were cultured in new incubators with $>$ two months of use, were collected for analyzing embryo development status during the 3-d culture. The grade I embryo rate, the cleavage rate, and the development arrest rate were also analyzed. Although there was no significant difference in cleavage rate between the new incubator early phase group and the control group (96.7\% vs $99.4 \%$ ), there was a significant decrease in the grade I embryo rate $(24.0 \%$ vs $40.5 \%)$, and an increase in the degradation rate (23.3\% vs $11.9 \%)$ and the development arrest rate $(6.7 \%$ vs $1.2 \%)$ in the new incubator early phase group. However, no differences were found in cleavage rate $(98.7 \%$ vs $99.4 \%)$, grade I embryo rate $(36.2 \%$ vs $40.5 \%)$, degradation rate $(10.5 \%$ vs $11.9 \%)$, and development arrest rate $(3.9 \%$ vs $1.2 \%)$ between the new incubator later phase group and the control group (Table 3). Taken together, the longer the incubators were used, the greater the grade I embryo rate, while the degradation rate and the development arrest rate decreased.

\section{Discussion}

It is hypothesized that the formation of 3PN zygotes is likely associated with a high sperm concentration, ${ }^{7}$ the abnormal maturity of eggs, and abnormal cortical granules. ${ }^{8}$ However, the detailed mechanisms are still elusive. Delivered fetuses from the very few 3PN embryos that survive often have severe congenital malformations. Therefore, 3PN embryos are not transferred in clinical practice and are usually discarded. However, they are excellent resources for chromosome studies of early-stage human embryos. Our study showed that $3 \mathrm{PN}$ zygotes had the same ability to cleave as $2 \mathrm{PN}$ zygotes.

The IVF laboratory environment directly affects the outcome of ART. In the female uterus, embryos develop in a protective, hypoxic, and light-free microenvironment with constant temperature ${ }^{9}$ and humidity. ${ }^{10}$ However, in ART,

Table 3 Development of 3PN Zygotes in the New and Old Incubators

\begin{tabular}{|l|c|c|c|}
\hline Index & $\begin{array}{c}\text { Old Incubator Group } \\
(\mathbf{n}=168)\end{array}$ & $\begin{array}{c}\text { New Incubator Early Phase Group } \\
(\mathbf{n}=150)\end{array}$ & $\begin{array}{c}\text { New Incubator Later Phase Group } \\
(\mathbf{n}=152)\end{array}$ \\
\hline Cleavage rate & $99.4 \%(167 / 168)$ & $96.7 \%(145 / 150)$ & $98.7 \%(150 / 152)$ \\
Grade I embryo rate & $40.5 \%(68 / 168)$ & $24.0 \%(36 / 150)^{a}$ & $36.2 \%(55 / 152)$ \\
Degradation rate & $11.9 \%(20 / 168)$ & $23.3 \%(35 / 150)^{a}$ & $10.5 \%(16 / 152)$ \\
Development arrest rate & $1.2 \%(2 / 168)$ & $6.7 \%(10 / 150)^{a}$ & $3.9 \%(6 / 152)$ \\
\hline
\end{tabular}

Note: ${ }^{a}$ By comparing with the old incubator groups. 
embryos develop in vitro and have to adapt to changes in temperature, humidity, $\mathrm{PH},{ }^{11}$ and osmolarity. ${ }^{12}$ All these factors can weaken embryo developmental potential. As such, the operating environment within the IVF laboratory must be strictly controlled. Compared with old IVF laboratories, embryos cultured within new IVF laboratories will face more serious environmental challenges in the form of building materials, interior decorating materials, and office supplies. ${ }^{13}$ The introduction of new incubators, new workstations, and new staff into newly built laboratories also contribute to instability of the in vitro process. Before a new laboratory is put into use, it is necessary to perform a quality test covering environment indicators, instruments, and various reagents and consumables. ${ }^{13}$ ART cannot take place unless all these tests are passed. ${ }^{14}$ At present, the most widely used environment quality control method in IVF laboratories is the mouse embryo biological test. ${ }^{15}$ Mouse embryos and human embryos share similar growth and development processes, which allows the use of mouse embryos in quality tests during each ART procedure. ${ }^{16}$ To ensure that any products used for embryo culture are not embryotoxic, they are tested with bioassays using animal embryos or human sperm instead of human embryos. But it is unknown whether any of these assays have similar sensitivity to human embryos under in vitro conditions. ${ }^{17}$ On the other hand, owing to the species difference, the sensitivity of mouse embryos to the environment has been widely questioned. ${ }^{18}$ Therefore, the mouse embryo test should not be regarded as the only indicator for laboratory culture systems, and it needs to be considered together with other indicators. After the new IVF laboratory at our center passed its environment test and mouse embryo biological test, we retrospectively analyzed the development of 168 3PN zygotes cultured in the old embryo laboratory and $1643 \mathrm{PN}$ zygotes cultured in the new laboratory after six months of use. The cleavage rate, grade I embryo rate, degeneration rate, and development arrest rate of the two groups were compared. The results showed that the development of the 3PN zygotes cultured in the new and old laboratories were consistent with those of the mouse embryo biological test, and that 3PN embryos could reflect the culture environment of the new IVF laboratory. Validation of the quality control of the new laboratory revealed no significant difference in pregnancy outcomes between the old and new laboratories, the data of which are going to be published separately.

Quality control of the new instruments and new laboratory was attempted in this study using human 3PN zygotes. The results showed that the grade I embryo rate, degeneration rate, and development arrest rate of 3PN zygotes cultured in the new instruments within the first two months of use changed significantly; however, after two months, the incubators were suitable for embryo culture. These findings demonstrate the utility of culturing human 3PN zygotes during the quality control of new instruments. There is a general recommendation for the use of more than $1003 \mathrm{PN}$ embryos. According to the the results of the current study, it is recommended that the human tripronuclear (3PN) zygote biological test be used. The cleavage rate should be $>98 \%$, the rate of high-quality embryos should be $>36 \%$, the degradation rate should be $<11 \%$, and the growth arrest rate should be $<4 \%$.

This method can be named the "human tripronuclear zygote biological test," which can also be applied to testing culture media and consumables. In conclusion, 3PN zygotes can be used for quality control within the IVF laboratory.

\section{Data Sharing Statement}

All datasets generated for this study are included in the article materials.

\section{Ethics Statements}

This study abided by the ethical standards of the Helsinki Declaration and national legislation and was approved by the Medical Ethical Committee of the Fujian Maternity and Child Health Hospital. The patients donated their tripronuclear (3PN) zygotes for research and signed informed consent forms.

\section{Acknowledgments}

This work was supported by Fujian Natural Science Foundation (2019J01511), Fujian Provincial Health Technology Project (NO.2020GGB016), Fujian Natural Science Foundation (2021J0122), and the Technology Innovation Startup Fund of Fujian Maternity and Child Health Hospital (YCXY20-02), Health Research Project of Department of Finance (Fujian finance refers to [2020] NO. 467), Health Research Project of Department of Finance (Fujian finance refers to [2020] NO. 827). 


\section{Disclosure}

The authors report no conflicts of interest.

\section{References}

1. Brazil C, Swan SH, Tollner CR, et al. Quality control of laboratory methods for semen evaluation in a multicenter research study. $J$ Urol. 2004;25 (4):645-656.

2. Swain JE. Decisions for the IVF laboratory: comparative analysis of embryo culture incubators. Reprod Biomed Online. 2014;28(5):535-547. doi:10.1016/j.rbmo.2014.01.004

3. Lane M, Mitchell M, Cashman KS, et al. To QC or not to QC: the key to a consistent laboratory? Reprod Fertil Dev. 2007;20(1):23-32. doi:10.1071/RD07161

4. Esfandiari N, Gubista A. Mouse embryo assay for human in vitro fertilization quality control: a fresh look. J Assist Reprod Genet. $2020 ; 37: 4131$.

5. Esteves SC, Bento FC. Air quality control in the ART laboratory is a major determinant of IVF success. Asian J Androl. 2016;18(4):596. doi:10.4103/1008-682X.166433

6. Munné S, Cohen J. Chromosome abnormalities in human embryos. Hum Reprod Update. 1998;4(6):842-855. doi:10.1093/humupd/4.6.842

7. Wolf D, Byrd W, Dandekar P, et al. Sperm concentration and the fertilization of human eggs in vitro. Biol Reprod. 1984;31(4):837-848. doi:10.1095/biolreprod31.4.837

8. Pellestor F, Andréo B, Anahory T, et al. The occurrence of aneuploidy in human: lessons from the cytogenetic studies of human oocytes. Eur J Med Genet. 2006;49(2):103-116. doi:10.1016/j.ejmg.2005.08.001

9. Fujiwara M, Takahashi K, Izuno M, et al. Effect of micro-environment maintenance on embryo culture after in-vitro fertilization: comparison of top-load mini incubator and conventional front-load incubator. J Assist Reprod Genet. 2007;24(1):5-9. doi:10.1007/s10815-006-9088-3

10. Calzi F, Papaleo E, Rabellotti E, et al. Exposure of embryos to oxygen at low concentration in a cleavage stage transfer program: reproductive outcomes in a time-series analysis. Clin Lab. 2012;58(9-10):997-1003.

11. Swain J. Is there an optimal pH for culture media used in clinical IVF? Hum Reprod Update. 2012;18(3):333-339. doi:10.1093/humupd/dmr053

12. Goto Y, Noda Y, Mori T, et al. Increased generation of reactive oxygen species in embryos cultured in vitro. Free Radic Biol Med. 1993;15 (1):69-75. doi:10.1016/0891-5849(93)90126-F

13. Mortimer D, Cohen J, Mortimer S, et al. Cairo consensus on the IVF laboratory environment and air quality: report of an expert meeting. Reprod Biomed Online. 2018;36(6):658-674. doi:10.1016/j.rbmo.2018.02.005

14. Gardner DK, Reed L, Linck D, et al. Quality control in human in vitro fertilization. Paper read at Seminars in Reproductive Medicine; 2005.

15. Ackerman S, Taylor S, Swanson R, et al. Mouse embryo culture for screening in human IVF. Arch Androl. 1984;12:129-136.

16. Gardner DK, Weissman A, Howles CM, et al. Textbook of Assisted Reproductive Techniques: Laboratory and Clinical Perspectives. CRC press; 2016.

17. Khan Z, Wolff HS, Fredrickson JR, et al. Mouse strain and quality control testing: improved sensitivity of the mouse embryo assay with embryos from outbred mice. Fertil Steril. 2013;99(3):847-854. doi:10.1016/j.fertnstert.2012.10.046

18. Hughes PM, Morbeck DE, Hudson SB, et al. Peroxides in mineral oil used for in vitro fertilization: defining limits of standard quality control assays. J Assist Reprod Genet. 2010;27(2-3):87-92. doi:10.1007/s10815-009-9383-x

International Journal of General Medicine

\section{Dovepress}

\section{Publish your work in this journal}

The International Journal of General Medicine is an international, peer-reviewed open-access journal that focuses on general and internal medicine, pathogenesis, epidemiology, diagnosis, monitoring and treatment protocols. The journal is characterized by the rapid reporting of reviews, original research and clinical studies across all disease areas. The manuscript management system is completely online and includes a very quick and fair peer-review system, which is all easy to use. Visit http://www.dovepress.com/testimonials.php to read real quotes from published authors.

Submit your manuscript here: https://www.dovepress.com/international-journal-of-general-medicine-journal 